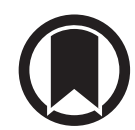

CrossMark

\title{
Pulmonary arterial hypertension: the burden of disease and impact on quality of life
}

\author{
Marion Delcroix ${ }^{1}$ and Luke Howard ${ }^{2}$
}

Affiliations: 'Dept of Respiratory Diseases, KU Leuven, University of Leuven and University Hospitals Leuven, Leuven, Belgium. ${ }^{2}$ Dept of Cardiac Sciences, Hammersmith Hospital and Imperial College London, London, UK.

Correspondence: Marion Delcroix, Dept of Respiratory Diseases, KU Leuven, University of Leuven and University Hospitals Leuven, Herestraat 49, B-3000 Leuven, Belgium. E-mail: marion.delcroix@uzleuven.be

ABSTRACT Pulmonary arterial hypertension (PAH) is a debilitating disease that pervades all aspects of a patient's daily life. It is also increasingly acknowledged that the burden of PAH extends to older patients and carers. Until recently, the adverse effect of disease symptoms on the physical, emotional and social factors governing patient health-related quality of life (HRQoL) remained largely unrecognised. With a shift in therapeutic objectives to longer term improvements and HRQoL benefits, clinical trials now frequently include HRQoL measures as study end-points. Most HRQoL instruments used in patients with $\mathrm{PAH}$ are generic or non-disease-specific questionnaires and therefore may not accurately capture PAH disease burden. New PAH-specific HRQoL instruments currently undergoing validation include emPHasis-10 and Pulmonary Arterial Hypertension-Symptoms and Impact (PAH-SYMPACT; Actelion Pharmaceuticals Ltd, Allschwil, Switzerland). Using various HRQoL measures, pharmacological therapies have been shown to improve HRQoL in patients with PAH. Patients also derive HRQoL benefits from nonpharmacological strategies, which include the emotional support provided by multidisciplinary care and support groups that is fundamental to patient wellbeing. Looking to the future, validated PAH-specific HRQoL instruments together with dedicated guidelines and procedures are essential to support the translation of HRQoL scores to the clinic, thus enabling a holistic treatment approach to the management of patients with PAH.

@ERSpublications

Clinical care and research in PAH should increase its focus on health-related quality of life http://ow.ly/Tcrtq

\section{Introduction}

Pulmonary arterial hypertension (PAH), characterised by increased pulmonary vascular resistance and arterial pressure, affects an estimated 15-60 per million of the population, and is more commonly diagnosed in women [1-3]. PAH causes a range of nonspecific symptoms (including breathlessness, fatigue, chest pain and weakness) $[3,4]$ and is associated with significant morbidity and mortality triggered by the debilitating progressive nature of the disease, which eventually leads to right heart failure and death [3,5-8]. The effect of disease symptoms on the patient's physical mobility and emotional state adversely affects health-related quality of life (HRQoL) $[9,10]$. Although pharmacological treatments for $\mathrm{PAH}$ (endothelin receptor antagonists, soluble guanylate cyclase stimulators or phosphodiesterase-5 inhibitors (PDE-5i), and prostacyclin analogues) and lung transplantation are available to alleviate disease symptoms and slow disease progression [3], treatment-related adverse events and the inconvenience and

Received: Aug 212015 | Accepted after revision: Sept 232015

Conflict of interest: Disclosures can be found alongside the online version of this article at err.ersjournals.com

Provenance: Publication of this peer-reviewed article was sponsored by Actelion Pharmaceuticals Ltd, Allschwil, Switzerland (principal sponsor, European Respiratory Review issue 138).

Copyright OERS 2015. ERR articles are open access and distributed under the terms of the Creative Commons Attribution Non-Commercial Licence 4.0. 
side-effects of some routes of drug administration (for example intravenous and subcutaneous delivery) can negatively influence patients' daily life [11].

For patients living with $\mathrm{PAH}$, the disease can be hugely devastating and exert an adverse impact on all aspects of life: physical, social and emotional $[4,11,12]$. However, despite the significant progress that has been made in the development of new therapies for PAH in the past two decades, the impact the disease has on patients' HRQoL was less well understood until recently. This article reviews the burden of PAH on patients and carers and outlines measures currently used to evaluate HRQoL. Management options for improving HRQoL in patients with $\mathrm{PAH}$, with a focus on available pharmacological and nonpharmacological strategies, are also discussed.

\section{The burden of PAH}

$\mathrm{PAH}$ imposes a considerable burden on the patient by affecting all aspects of daily life that govern HRQoL, from physical activity, general health and vitality to emotional and social functioning (figure 1) $[9,12]$. Furthermore, when PAH is associated with conditions such as systemic sclerosis, patients have to contend with the additional HRQoL burden of the primary condition [12]. Overall, the debilitating impact of PAH on HRQoL is clear when compared with other medical conditions, being at least as severe as illnesses such as chronic obstructive pulmonary disease and renal failure (figure 1), and those conditions well recognised as being severely debilitating and life-threatening such as spinal cord injury, interstitial lung disease or treatment-resistant cancer [12-15].

In particular, the physical activity of many individuals is reported to be severely affected by $\mathrm{PAH}$ symptoms of breathlessness, fatigue and lack of energy, which, in turn, restrict the completion of even the smallest everyday tasks such as housework and food shopping [4]. With respect to specific physical activities that are affected by the disease, the International Patient and Carer Report from the European Pulmonary Hypertension Association (PHA) revealed that the majority (83\%) of the 326 surveyed patients with PAH experienced difficulty climbing stairs and almost all patients (97\%) found that the disease affected their participation in sports and exercise to some extent [4]. These European findings are mirrored by qualitative reports from the Patient Voice survey ( $~ 85$ participants) initiated by the US Food and Drug Administration (FDA), which found that commonly reported symptoms of breathlessness and fatigue were similarly restrictive on the daily physical activities of patients [11]. Results from other studies suggest that the reduced physical activity due to $\mathrm{PAH}$, as identified in the European PHA and FDA Patient Voice surveys [4, 11], has negative implications for long-term outcomes. A recent study used a wrist-worn device (actigraph) to monitor the everyday physical performance of patients with PAH or chronic thromboembolic pulmonary hypertension over a 2-week period who were then followed-up for $\geqslant 4$ years [7]. The study authors concluded that reduced activity $(<15 \mathrm{~h}$ of activity during the day) and a

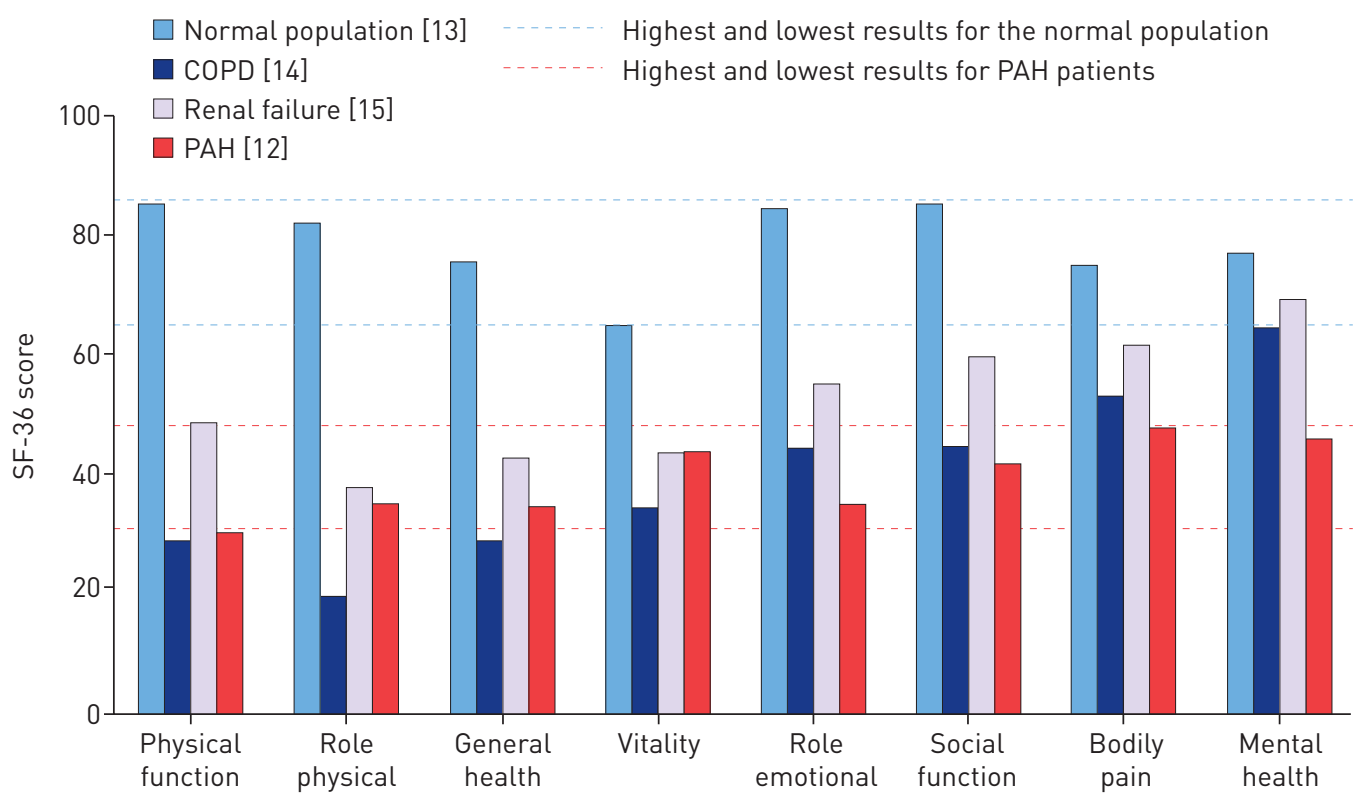

FIGURE 1 Effect of pulmonary arterial hypertension (PAH) on SF-36-measured health-related quality of life (HRQoL) measures versus the normal population and other disease conditions [12-15]. COPD: chronic obstructive pulmonary disease; SF-36: Medical Outcomes Study 36-item short form. 
prolonged nocturnal rest correlated with severe haemodynamic impairment, reduced transplantation-free survival and overall poorer prognosis [7]. The study also demonstrated that daytime activity positively correlated with HRQoL [7], which is consistent with observations that functional status is a stronger determinant of HRQoL than haemodynamic values [12]. Thus, as would be expected, with disease progression, the decline in physical function reduces HRQoL in patients with PAH $[9,12]$.

While the physical consequences of PAH are widely acknowledged, the psychological impact of PAH is frequently overlooked by patients and clinicians [16, 17]. Feelings of social isolation are often expressed by patients with $\mathrm{PAH}$, who ascribe this to the fact that there are no overt signs of their condition (i.e. the disease is not "visible") and to a lack of understanding or knowledge about the disease in the community and among their friends and family [4]. These feelings are particularly prominent among patients aged $<30$ years [4], an age group for whom social interaction may be a particularly significant part of their daily life. Aside from social isolation, the impact of PAH can be seen on broader aspects of psychological health. In a study of 101 patients with $\mathrm{PAH}$, almost half (48\%) suffered from mild to extremely severe symptoms of anxiety, and approximately one-third experienced depressive (32.6\%) and stress (27.6\%) symptoms [16]. An earlier report, claiming to be the first study to investigate the prevalence of psychiatric disorders in patients with pulmonary hypertension $(\mathrm{PH})$, identified a concomitant psychiatric disorder in more than one-third (35.4\%) of patients [17]. In the study population, major depressive disorder and panic disorders were the most common psychiatric disorders [17]. Interestingly, the prevalence of major depressive disorder and panic disorder increased with greater functional impairment in patients participating in the study [17]. Among those patients with a psychiatric diagnosis, only $24 \%$ received psychiatric treatment, most commonly psychopharmacological medications [17]. Despite such studies, the association between $\mathrm{PAH}$ and comorbid psychological symptoms (e.g. depression) continues to be under-recognised, as does the impact of these symptoms on the HRQoL of patients with PAH $[6,16]$.

In an attempt to address this evidence gap, a recent study examined the prevalence of psychological symptoms and their association with HRQoL in patients with PAH. The researchers noted that depressive symptoms, together with functional class and disability, had a major impact on the observed variance in HRQoL values among the study population [16].

Not only are there physical and psychological consequences for patients with $\mathrm{PAH}$, employment is also adversely affected by the disease and can lead to a significant reduction in household income [4]. In the European PHA survey, 85\% of participating patients reported that their work was affected by the disease [4]. Of the $48 \%$ of patients who had to give up work or needed help in order to maintain employment, most $(73 \%)$ experienced a loss in average household income, with one in six reporting that their household income had more than halved [4]. In addition, more than one-third (35\%) of carers reported a reduction in income because of a decrease in work hours or having to give up work completely to care for their relative with $\mathrm{PAH}[4]$.

While surveys such as the European PHA and US Patient Voice reports have highlighted the significant impact of PAH on the general population of patients $[4,11]$, there is growing recognition among clinicians that the burden of PAH may be particularly significant among older populations [1]. PAH is increasing in prevalence in patients aged 50-65 years, who are more likely to be diagnosed in advanced stages of the disease (World Health Organization functional class (WHO FC) III/IV), have lower exercise capacity and a greater comorbidity burden [1]. Comorbid ischaemic heart disease, coronary artery disease, hypertension, atrial fibrillation, diabetes and hypothyroidism have all been reported at higher rates in patients with PAH aged $>50$ years compared with younger patients $[1,18]$. The presence of multiple comorbidities often leads to a delay in the diagnosis of PAH in older patients, which may account for the relatively low level of disease recognition in this population in earlier years [1]. This higher comorbidity burden may also contribute to the poorer survival rates seen among a cohort of newly diagnosed older patients in the UK and Ireland who were three-fold more likely to die versus their younger counterparts ( $\leqslant 50$ years of age); the latter group had fewer comorbidities and better functional and exercise capacity [19].

Women with PAH of child-bearing age face the additional burden of a significantly increased risk of complications during pregnancy as a result of their poor tolerance of the associated haemodynamic and physiological changes, which can lead to right ventricle failure and arrhythmias [20]. Pregnancy is associated with a substantial mortality rate in PAH [21], although there has been evidence to show that survival among pregnant women with PAH has improved over the past few decades [22]. Nevertheless, it is recommended that patients with PAH avoid pregnancy [3]. The contraindication for pregnancy can have a detrimental effect on family life for patients with $\mathrm{PAH}$ [23], and the fear of becoming pregnant can also have a negative impact on patients' relationships with their partners [4].

The burden of PAH not only affects the patients, but also extends to their carers [4]. Until studies such as the European PHA and US FDA Patient Voice surveys were conducted, research on the impact of PAH on 
patient life was scarce $[4,11]$. Therefore, it is unsurprising that there is a similar lack of research regarding the effect of PAH on carers, a role often taken by the patient's partner or family members [4]. The aforementioned European PHA survey, in addition to patients, included 129 carers whose relationship to the patient (not necessarily a patient included in the survey) was either as a spouse or partner (55\%), parent $(21 \%)$, child $(18 \%)$, family member $(5 \%)$ or friend (1\%) [4]. More than half (57\%) of carers reported a very high impact (rating of $8-10$ on a scale of 10 ) from the patient's PAH on their own daily lives, with the proportion of carers experiencing this level of impact on their life increasing as the patient's functional status declined (41\%, 53\% and $88 \%$ of those caring for a patient with WHO FC II, III and IV, respectively). A significant proportion of carers (43\%) found the extra tasks and responsibilities associated with the patient's condition physically exhausting [4]. Moreover, feelings of social isolation and disrupted sexual relationships experienced by patients were also reported by carers, in addition to other changes in carers' emotional and social wellbeing [4]. A further caregiver study noted the perceived lack of social support reported by a surveyed population of 35 carers of patients with PAH [24]. The study also found a significant association between lower levels of social support and greater severity of depressive symptoms in carers [24]. On a positive note, the overwhelming majority (92\%) of carers in the European PHA survey felt that they were helping to improve the patient's HRQoL [4].

For patients with $\mathrm{PAH}$, and by extension their carers, pharmacological treatments form the mainstay of disease management. However, invasive, cumbersome and painful routes of administration for some treatments (e.g. parenteral, subcutaneous or inhaled prostacyclin therapy) and side-effects of therapy can adversely affect treatment acceptance and HRQoL in patients with PAH [11, 25-28]. As reported by participants in the FDA Patient Voice survey, daily life is significantly affected by the burden of living with an intravenous line, while the complexity and inconvenience of some drug regimens can be difficult to incorporate into everyday life [11].

Additionally, intolerable side-effects such as site infections from intravenous lines or the pain of subcutaneous treatment were cited in the Patient Voice survey as reasons for poor treatment acceptance and changes in treatment [11]. The requirement for oxygen therapy is also associated with poor HRQoL [9]. Although patients can adjust to oxygen use, adherence can be affected by the patient's perception of the interference caused by the restrictions of oxygen dependence [9]. The development of oral formulations of PAH therapies (such as endothelin receptor antagonists, PDE-5i and prostacyclin analogues) and a room temperature stable intravenous formulation of epoprostenol containing arginine and sucrose may improve treatment acceptance through greater convenience, compared with existing intravenous formulations [28-31].

\section{Overview of HRQoL questionnaires}

As the number of PAH treatment options has expanded, clinical outcomes have improved beyond short-term functional and symptomatic benefits. Therefore, the objectives of therapy are now focused on longer term improvements and beneficial effects on HRQoL [25, 32]. Consequently, measurement of HRQoL is increasingly incorporated within the design of PAH clinical trials as a secondary or exploratory end-point [25]. HRQoL measures fall within the category of health status measures defined as patient-reported outcomes (PROs), which refer to measurements that are directly obtained from the patient without influence or the interpretation of response from others [33, 34]. The use of PRO instruments is advocated to assess specific variables that are best known by the patient or best measured from the patient's perspective (for example, symptoms or their impact) [34].

Various HRQoL instruments have been employed to measure the effects of PAH on patients' daily lives, the majority of which are generic or non-PAH-specific measures (table 1) [33, 35-41]. HRQoL assessments are administered in the form of a questionnaire, but there are inherent differences between scales, including the length of the questionnaire and health domains evaluated (table 1). Furthermore, depending on the HRQoL scale, a higher score can be indicative of either better or worse HRQoL (figure 2). HRQoL validity analyses suggest that existing generic scales are not wholly ideal for use in patients with PAH. For example, the Medical Outcomes Study 36-item short form (SF-36) and the Minnesota Living with Heart Failure Questionnaire correlate poorly with haemodynamic measurements [33], which emphasises the fact that non-PAH-specific instruments may not accurately capture the PAH burden and may be less sensitive to change in HRQoL with treatment [25].

A growing understanding of the limitations of existing HRQoL scales, together with the need for standardisation of HRQOL measures in $\mathrm{PAH}$, has focused research efforts towards developing and validating PAH-specific instruments. At present, Cambridge Pulmonary Hypertension Outcome Review (CAMPHOR) represents the only validated questionnaire for evaluation of HRQoL in patients with $\mathrm{PH}$ [42], but the questionnaire may have limited application in routine clinical practice given its length (65 items), cost, and the fact that it has only been validated for 11 languages [43]. Furthermore, despite its focus 
TABLE 1 Summary of health-related quality of life scales used in pulmonary arterial hypertension

\begin{tabular}{|c|c|c|c|}
\hline Scale & Domains & Items $\mathbf{n}$ & Recall period \\
\hline \multicolumn{4}{|l|}{ Generic } \\
\hline SF-36 [35] & $\begin{array}{c}8 \text { domains: physical functioning, role physical, bodily pain, general health, } \\
\text { vitality, social functioning, role emotional, mental health }\end{array}$ & 36 & Now to past 4 weeks \\
\hline$E Q-5 D[36]$ & 5 domains: mobility, self-care, usual activities, pain/discomfort, anxiety/depression & 5 & Today \\
\hline NHP [37] & $\begin{array}{c}6 \text { domains: physical mobility, pain, social isolation, } \\
\text { emotional reactions, energy level, sleep }\end{array}$ & 38 & At the moment \\
\hline \multicolumn{4}{|l|}{ Disease-specific } \\
\hline MLHFQ [39] & 2 domains: physical, emotional & 21 & 4 weeks \\
\hline $\mathrm{LPH}^{\#}[40]$ & 2 domains: physical, emotional & 21 & 1 week \\
\hline $\mathrm{CHQ}[41]$ & 4 domains: dyspnoea, fatigue, emotional function, mastery & 20 & 2 weeks \\
\hline emPHasis-10 [42] & Unidimensional & 10 & At the moment \\
\hline
\end{tabular}

SF-36: Medical Outcomes Study 36-item short form; EQ-5D: EuroQol Group 5-Dimension Self-Report Questionnaire; NHP: Nottingham Health Profile; CAMPHOR: Cambridge Pulmonary Hypertension Outcome Review; MLHFQ: Minnesota Living with Heart Failure Questionnaire; LPH: Living with Pulmonary Hypertension questionnaire; CHQ: Chronic Heart Failure Questionnaire. " : adapted from MLHFQ. Information from [25, 33].

on the PH population, CAMPHOR may not accurately encapsulate all the key PAH symptoms [40, 42]. Accordingly, researchers have placed greater emphasis on designing a simple, rapidly administered disease-specific questionnaire that is easy to use in clinical practice for evaluation of HRQoL in patients with PAH [42]. The PAH-specific PRO instrument Pulmonary Arterial Hypertension-Symptoms and Impact (PAH-SYMPACT; Actelion Pharmaceuticals Ltd, Allschwil, Switzerland) questionnaire to assess PAH symptoms and their impact on patients' lives is currently being developed in accordance with the US FDA guidance on PRO development [44]. Based on qualitative research in patients with $\mathrm{PAH}$, a draft conceptual framework for the PAH-SYMPACT questionnaire was developed, encompassing four symptom domains (respiratory symptoms, tiredness, cardiovascular symptoms and other symptoms) and five impact domains (physical activities, daily activities, social impact, cognition and emotional impact) [44]. The PAH-SYMPACT tool is currently undergoing final content and psychometric validation in phase $3 \mathrm{~b}$, prospective, single-arm, open-label studies in patients with PAH in the USA (SYMPHONY; NCT01841762) and Europe (ORCHESTRA; NCT02081690) [44-46]. Another recently proposed PAH-specific tool is emPHasis-10, a 10-item questionnaire, which in a qualitative study of 226 patients with $\mathrm{PH}$ ( $82 \%$ of whom had $\mathrm{PAH}$ ) was reported to demonstrate sensitivity to clinical parameters relevant to $\mathrm{PAH}$, such as change in WHO FC [42].

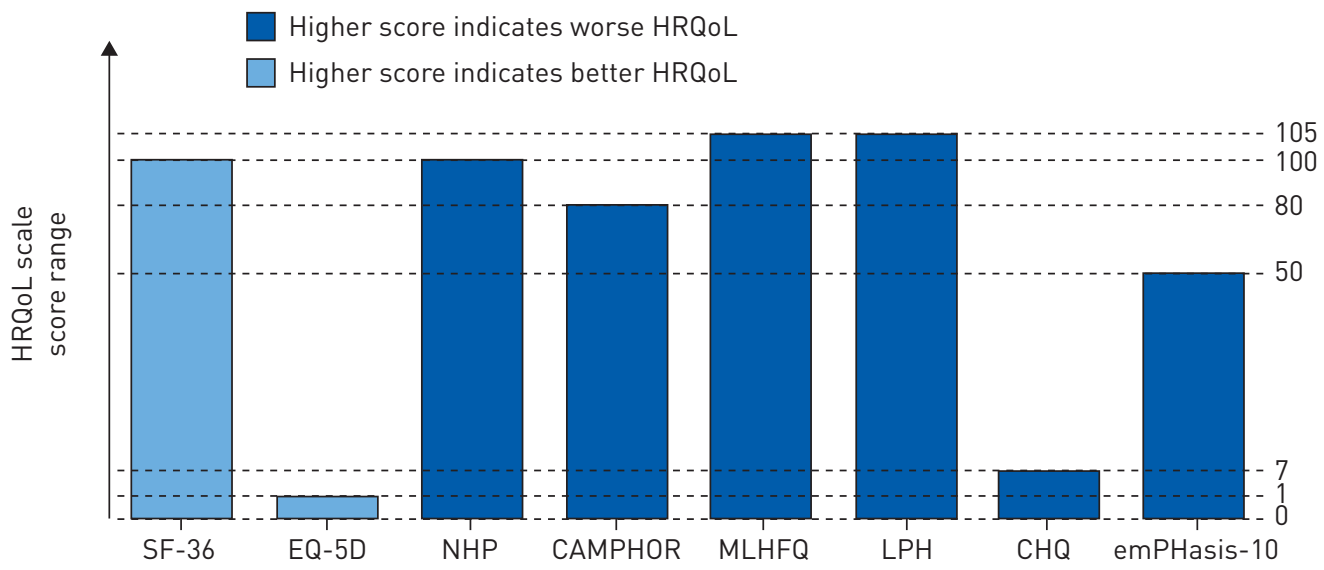

FIGURE 2 Scoring system for health-related quality of life (HRQoL) scales [33, 40]. SF-36: Medical Outcomes Study 36-item short form; EQ-5D: EuroQol Group 5-Dimension Self-Report Questionnaire; NHP: Nottingham Health Profile; CAMPHOR: Cambridge Pulmonary Hypertension Outcome Review; MLHFQ: Minnesota Living with Heart Failure Questionnaire; LPH: Living with Pulmonary Hypertension questionnaire; CHQ: Chronic Heart Failure Questionnaire. 


\section{PAH management: improving HRQoL}

Clinical studies have shown that PAH therapies improve HRQoL in patients with PAH [47-57]. In these studies, various scales were employed to evaluate change in HRQoL, which limits comparisons between studies [33]. Nevertheless, the SF-36 (alone or in combination with the EuroQol Group 5-Dimension Self-Report Questionnaire (EQ-5D)) was the most commonly used measure across trials [25, 58]. Early studies of PDE-5i, Pulmonary Arterial Hypertension and Response to Tadalafil (PHIRST) and Sildenafil Use in Pulmonary Arterial Hypertension 1 (SUPER-1), measured change in HRQoL for $\leqslant 24$ weeks of treatment in patients with PAH via the SF-36 and EQ-5D $[55,56]$. Results from these two studies reported significant improvements in six of eight SF-36 domains and EQ-5D scores with tadalafil $(\mathrm{p}<0.05)$, while physical functioning, vitality and general health domains, and the EQ-5D health status and utility index scores were significantly improved in patients who received sildenafil $(p<0.05)[55,56]$. In the Ambrisentan in Pulmonary Arterial Hypertension, Randomized, Double-Blind, Placebo-Controlled, Multicenter, Efficacy Study (ARIES)-1, the endothelin receptor antagonist ambrisentan was associated with significant improvement in the SF-36 physical functioning domain at 12 weeks $(p=0.005)$, whereas a trend towards improvement in SF-36-measured health domains did not reach statistical significance in ARIES-2 [47]. More recently, the long-term Study with an Endothelin Receptor Antagonist in Pulmonary Arterial Hypertension to Improve Clinical Outcome (SERAPHIN) trial showed that macitentan significantly improved seven of eight health domains and both the physical and mental component summary scores of the SF-36 at month $6(\mathrm{p}<0.05)$ [50]. Given the treatment-related benefits in HRQoL observed in patients with $\mathrm{PAH}$, mounting evidence implicates a future role for improved HRQoL as a prognostic marker in $\mathrm{PAH}$ [59]. In a study of 54 consecutive newly diagnosed patients with PAH who received first-line endothelin receptor antagonists or PDE-5i, a baseline SF-36 Physical Component Summary (PCS) score $>32$ was associated with a better survival rate than a score $<32(p=0.04)$. Moreover, patients exhibiting a PCS score $\geqslant 38$ after 16 weeks of treatment demonstrated superior 3-year survival rates versus those who scored $<38$ on the PCS ( $\mathrm{p}=0.016)$ [59].

In addition to these PAH-targeted pharmacological treatments, agents used for the management of concomitant conditions (e.g. for depression or poor sleep quality) play an essential role in improving patient comfort, which, in turn, has the potential to exert a significant positive impact on HRQoL [60]. Furthermore, nonpharmacological strategies such as exercise training and palliative care can prove beneficial for improving HRQoL in patients with PAH [10, 61-63]. Symptoms of PAH that affect physical function have a negative impact on HRQoL [10]. It follows, therefore, that improvements in physical fitness and function through exercise rehabilitation can positively affect patient HRQoL [10]. Indeed, some clinical evidence supports the beneficial effects of exercise training on patient HRQoL in addition to increases in aerobic capacity, muscle strength and exercise tolerance [61]. However, further studies are necessary to conclusively support this finding [10].

Although palliative care, such as end-of-life management by a multidisciplinary healthcare team and/or hospice referral, is another nonpharmacological strategy that may confer significant HRQoL benefits to patients, surveys suggest that patients may be unaware of, or are not offered, the opportunity to explore this option [62-64].

Other nonpharmacological interventions may serve an important purpose in providing the emotional support that has been shown to be fundamental to the wellbeing of patients with $\mathrm{PAH}$ and their families [4]. Accordingly, the adequate provision of psychosocial support has undergone close scrutiny by professional organisations. In their recent guidance, the joint task force of the European Society of Cardiology (ESC) and the European Respiratory Society (ERS) issued a level I-C recommendation (recommended/indicated based on expert opinion and/or observational studies and registries) for patients to receive psychosocial support [3], reflecting the importance of this approach for improving HRQoL. Furthermore, the task force advised that patients and their families should be encouraged to join patient support groups. These organisations can have an important role in helping patients cope with their PAH and can improve their confidence and outlook on life [3]. The ESC/ERS guidelines state that multidisciplinary teams should be involved in the delivery of psychosocial support, so that the psychological, social, financial, emotional and spiritual needs of patients can be managed effectively [3]. In this respect, the multidisciplinary team can perform a vital role in assuming responsibility for the evaluation of patient HRQoL and translation of these results into clinical practice [65]. Ultimately, in attempting to delineate and reduce the burden of PAH on the patient, it is important to understand that physician and patient expectations from disease management are likely to differ [58].

\section{Conclusions}

The considerable burden of PAH and its impact on the HRQoL of both patients and their carers is becoming increasingly recognised. It is encouraging that recognition of the importance of patient quality of 
life is assuming prominence in the clinical setting in terms of greater emphasis on practice recommendations and implementation of HRQoL measures to evaluate treatment effectiveness. Nevertheless, areas of uncertainty remain, primarily the need for guidelines and procedures to support the translation of HRQoL scores to the clinic. Further developments should focus on the assessment of HRQoL based on validated PAH-specific PRO instruments, which is essential to gauge the impact of the disease on patients' HRQoL and thus facilitate a step towards better holistic treatment of patients with PAH.

\section{Acknowledgements}

The authors would like to thank Anusha Bolonna from PAREXEL (Worthing, UK) for medical writing assistance, funded by Actelion Pharmaceuticals Ltd (Allschwil, Switzerland).

\section{References}

1 Hoeper MM, Gibbs JSR. The changing landscape of pulmonary arterial hypertension and implications for patient care. Eur Respir Rev 2014; 23: 450-457.

2 Sanchez O, Marié E, Lerolle U, et al. Pulmonary arterial hypertension in women. Rev Mal Respir 2010; 27: e79-e87.

3 Galiè N, Humbert M, Vachiery JL, et al. 2015 ESC/ERS Guidelines for the diagnosis and treatment of pulmonary hypertension). Eur Heart J 2015 [in press; DOI: 10.1093/eurheartj/ehv317].

4 European Pulmonary Hypertension Association (PHA). The impact of pulmonary arterial hypertension (PAH) on the lives of patients and carers: results from an international survey. www.phaeurope.org/wp-content/uploads/ International-PAH-patient-and-Carer-Survey-Report-FINAL1.pdf Date last updated: September 2012. Date last accessed: June 29, 2015.

5 Humbert M, Sitbon O, Chaouat A, et al. Survival in patients with idiopathic, familial, and anorexigen-associated pulmonary arterial hypertension in the modern management era. Circulation 2010; 122: 156-163.

6 Verma S, Cardenas-Garcia J, Mohapatra PR, et al. Depression in pulmonary arterial hypertension and interstitial lung diseases. N Am J Med Sci 2014; 6: 240-249.

7 Ulrich S, Fischler M, Speich R, et al. Wrist actigraphy predicts outcome in patients with pulmonary hypertension Respiration 2013; 86: 45-51.

8 Corris P, Degano B. Severe pulmonary arterial hypertension: treatment options and the bridge to transplantation. Eur Respir Rev 2014; 23: 488-497.

9 Matura LA, McDonough A, Carroll DL. Health-related quality of life and psychological states in patients with pulmonary arterial hypertension. J Cardiovasc Nurs 2014; 29: 178-184.

10 Buys R, Avila A, Cornelissen VA. Exercise training improves physical fitness in patients with pulmonary arterial hypertension: a systematic review and meta-analysis of controlled trials. BMC Pulm Med 2015; 15: 40.

11 US Food and Drug Administration (FDA). The voice of the patient. A series of reports from the U.S. Food and Drug Administration's (FDA's) patient-focused drug development initiative. Pulmonary arterial hypertension. www.fda.gov/downloads/ForIndustry/UserFees/PrescriptionDrugUserFee/UCM429382.pdf Date last updated: December 2014. Date last accessed: August 17, 2015.

12 Taichman DB, Shin J, Hud L, et al. Health-related quality of life in patients with pulmonary arterial hypertension. Respir Res 2005; 6: 92.

13 Wight JP, Edwards L, Brazier J, et al. The SF36 as an outcome measure of services for end stage renal failure. Qual Health Care 1998; 7: 209-221.

14 Hopman WM, Towheed T, Anastassiades T, et al. Canadian normative data for the SF-36 health survey. Canadian Multicentre Osteoporosis Study Research Group. CMAJ 2000; 163: 265-271.

15 Harper R, Brazier JE, Waterhouse JC, et al. Comparison of outcome measures for patients with chronic obstructive pulmonary disease (COPD) in an outpatient setting. Thorax 1997; 52: 879-887.

16 Vanhoof JMM, Delcroix M, Vandevelde E, et al. Emotional symptoms and quality of life in patients with pulmonary arterial hypertension. J Heart Lung Transplant 2014; 33: 800-808.

17 Löwe B, Gräfe K, Ufer C, et al. Anxiety and depression in patients with pulmonary hypertension. Psychosom Med 2004; 66: 831-836.

18 Shimony A, Eisenberg MJ, Rudski LG, et al. Prevalence and impact of coronary artery disease in patients with pulmonary arterial hypertension. Am J Cardiol 2011; 108: 460-464.

19 Ling Y, Johnson MK, Kiely DG, et al. Changing demographics, epidemiology, and survival of incident pulmonary arterial hypertension: results from the pulmonary hypertension registry of the United Kingdom and Ireland. Am $J$ Respir Crit Care Med 2012; 186: 790-796.

20 Tabarsi N, Levy R, Rychel V, et al. Pregnancy among women with pulmonary arterial hypertension: a changing landscape? Int J Cardiol 2014; 177: 490-491.

21 Jaïs X, Olsson KM, Barbera JA, et al. Pregnancy outcomes in pulmonary arterial hypertension in the modern management era. Eur Respir J 2012; 40: 881-885.

22 Bédard E, Dimopoulos K, Gatzoulis MA. Has there been any progress made on pregnancy outcomes among women with pulmonary arterial hypertension? Eur Heart J 2009; 30: 256-265.

23 Tsangaris I. Improving patient care in pulmonary arterial hypertension: addressing psychosocial issues. J Clin Hypertens 2014; 16: 159-161.

24 Hwang B, Howie-Esquivel J, Fleischmann KE, et al. Family caregiving in pulmonary arterial hypertension. Heart Lung 2012; 41: 26-34.

25 Rival G, Lacasse Y, Martin S, et al. Effect of pulmonary arterial hypertension-specific therapies on health-related quality of life: a systematic review. Chest 2014; 146: 686-708.

26 de Jesus Perez VA, Rosenzweig E, Rubin LJ, et al. Safety and efficacy of transition from systemic prostanoids to inhaled treprostinil in pulmonary arterial hypertension. Am J Cardiol 2012; 110: 1546-1550.

27 Shafazand S, Goldstein MK, Doyle RL, et al. Health-related quality of life in patients with pulmonary arterial hypertension. Chest 2004; 126: 1452-1459. 

formulation of intravenous epoprostenol in patients with pulmonary arterial hypertension. Am Heart J 2014; 167: 210-217.

30 Subramaniyan G, Jain D, Lohiya B, et al. Recent advances in the management of pulmonary hypertension. Indian J Clin Pract 2014; 25: 414-415.

31 Safdar Z. Targeted oral therapies in the treatment of pulmonary arterial hypertension. Clin Drug Investig 2010; 30 : 811-826.

32 Mandras SA, Gilkin RJ Jr, Pruett JA, et al. Pulmonary arterial hypertension: progress and challenges in the modern treatment era. Am J Manag Care 2014; 20: Suppl. 9, S191-S199.

33 Chen H, Taichman DB, Doyle RL. Health-related quality of life and patient-reported outcomes in pulmonary arterial hypertension. Proc Am Thorac Soc 2008; 5: 623-630.

34 US Food and Drug Administration. Guidance for industry. Patient-reported outcome measures: use in medical product development to support labeling claims. www.fda.gov/downloads/Drugs/Guidances/UCM193282.pdf Date last updated: December 2009. Date last accessed: May 6, 2015.

35 Ware JE Jr, Sherbourne CD. The MOS 36-item short-form health survey (SF-36). I. Conceptual framework and item selection. Med Care 1992; 30: 473-483.

35 EuroQoL Group. EuroQol - a new facility for the measurement of health-related quality of life. Health Policy 1990; 16: 199-208.

37 Hunt SM, McEwen J. The development of a subjective health indicator. Sociol Health Illn 1980; 2: 231-246.

38 McKenna SP, Doughty N, Meads DM, et al. The Cambridge Pulmonary Hypertension Outcome Review (CAMPHOR): a measure of health-related quality of life and quality of life for patients with pulmonary hypertension. Qual Life Res 2006; 15: 103-115.

39 Rector TS, Kubo SH, Cohn JN. Patients' self-assessment of their congestive heart failure. Part 2: Content, reliability and validity of a new measure, the Minnesota Living with Heart Failure Questionnaire. Heart Fail 1987; 3: 198-209.

40 Bonner N, Abetz L, Meunier J, et al. Development and validation of the living with pulmonary hypertension questionnaire in pulmonary arterial hypertension patients. Health Qual Life Outcomes 2013; $11: 161$.

41 Guyatt GH, Nogradi S, Halcrow S, et al. Development and testing of a new measure of health status for clinical trials in heart failure. J Gen Intern Med 1989; 4: 101-107.

42 Yorke J, Corris P, Gaine S, et al. emPHasis-10: development of a health-related quality of life measure in pulmonary hypertension. Eur Respir J 2014; 43: 1106-1113.

43 Galen Research. Measures database. CAMPHOR - Language Adaptations Available. Available from: www.galenresearch.com/measures-database/ Date last accessed: October 22, 2015.

44 McCollister D, Kummer S, Badesch D, et al. Development of the Pulmonary Arterial Hypertension-Symptoms and Impact (PAH-SYMPACT) Questionnaire: a new disease-specific patient-reported outcome instrument for PAH. Am J Respir Crit Care Med 2013; 187: A3294.

45 ClinicalTrials.gov. SYMPHONY: A Study of Macitentan in Pulmonary Arterial Hypertension to Validate the PAH-SYMPACT. https://clinicaltrials.gov/ct2/show/NCT01841762 Date last updated: 2015. Date last accessed: July 2, 2015.

46 ClinicalTrials.gov. A Pulmonary Arterial Hypertension Study With Macitentan to Validate the PAH-SYMPACT ${ }^{\mathrm{TM}}$ in France, Italy and Spain (ORCHESTRA). https://clinicaltrials.gov/ct2/show/NCT02081690 Date last updated: 2015. Date last accessed: July 2, 2015.

47 Galiè N, Olschewski H, Oudiz RJ, et al. Ambrisentan for the treatment of pulmonary arterial hypertension: results of the ambrisentan in pulmonary arterial hypertension, randomized, double-blind, placebo-controlled, multicenter, efficacy (ARIES) study 1 and 2. Circulation 2008; 117: 3010-3019.

48 Galiè N, Rubin LJ, Hoeper M, et al. Treatment of patients with mildly symptomatic pulmonary arterial hypertension with bosentan (EARLY study): a double-blind, randomised controlled trial. Lancet 2008; 371: 2093-2100.

49 Girgis RE, Frost AE, Hill NS, et al. Selective endothelin A receptor antagonism with sitaxsentan for pulmonary arterial hypertension associated with connective tissue disease. Ann Rheum Dis 2007; 66: 1467-1472.

50 Mehta S, Channick RN, Delcroix M, et al. Macitentan improves health-related quality of life in pulmonary arterial hypertension: results from the randomized controlled SERAPHIN trial. Am J Respir Crit Care Med 2013; 187: A3269.

51 Barst RJ, Rubin LJ, Long WA, et al. A comparison of continuous intravenous epoprostenol (prostacyclin) with conventional therapy for primary pulmonary hypertension. N Engl J Med 1996; 334: 296-301.

52 Olschewski H, Simonneau G, Galiè N, et al. Inhaled iloprost for severe pulmonary hypertension. $N$ Engl J Med 2002; 347: 322-329.

53 Simonneau G, Barst RJ, Galiè N, et al. Continuous subcutaneous infusion of treprostinil, a prostacyclin analogue, in patients with pulmonary arterial hypertension: a double-blind, randomized, placebo-controlled trial. $\mathrm{Am} J$ Respir Crit Care Med 2002; 165: 800-804.

54 Sastry BK, Narasimhan C, Reddy NK, et al. Clinical efficacy of sildenafil in primary pulmonary hypertension: a randomized, placebo-controlled, double-blind, crossover study. J Am Coll Cardiol 2004; 43: 1149-1153.

55 Pepke-Zaba J, Gilbert C, Collings L, et al. Sildenafil improves health-related quality of life in patients with pulmonary arterial hypertension. Chest 2008; 133: 183-189.

56 Pepke-Zaba J, Beardsworth A, Chan M, et al. Tadalafil therapy and health-related quality of life in pulmonary arterial hypertension. Curr Med Res Opin 2009; 25: 2479-2485.

57 Ghofrani HA, Galiè N, Grimminger F, et al. Riociguat for the treatment of pulmonary arterial hypertension. N Engl J Med 2013; 369: 330-340.

58 Howard LS, Ferrari P, Mehta S. Physicians' and patients' expectations of therapies for pulmonary arterial hypertension: where do they meet? Eur Respir Rev 2014; 23: 458-468.

59 Fernandes CJ, Martins BC, Jardim CV, et al. Quality of life as a prognostic marker in pulmonary arterial hypertension. Health Qual Life Outcomes 2014; 12: 130. 
Batal O, Khatib OF, Bair N, et al. Sleep quality, depression, and quality of life in patients with pulmonary hypertension. Lung 2011; 189: 141-149.

61 Arena R, Cahalin LP, Borghi-Silva A, et al. The effect of exercise training on the pulmonary arterial system in patients with pulmonary hypertension. Prog Cardiovasc Dis 2015; 57: 480-488.

62 Fenstad ER, Shanafelt TD, Sloan JA, et al. Physician attitudes toward palliative care for patients with pulmonary arterial hypertension: results of a cross-sectional survey. Pulm Circ 2014; 4: 504-510.

63 Swetz KM, Shanafelt TD, Drozdowicz LB, et al. Symptom burden, quality of life, and attitudes toward palliative care in patients with pulmonary arterial hypertension: results from a cross-sectional patient survey. $J$ Heart Lung Transplant 2012; 31: 1102-1108.

64 Shipley J, Grinnan D, Swetz K, et al. The Health Care Initiative for Improving End of Life in Pulmonary Hypertension (CARING). Pulmonary Hypertension Association Online University. www.phaonlineuniv.org/ResourceLibrary/ Resource.cfm?ItemNumber=3680 Date last updated: September 22, 2011. Date last accessed: May 14, 2015.

65 Gihl A. Health-related quality of life in pulmonary arterial hypertension. Advances in PH 2010; 8: Winter 2010. 